\title{
Afrontamiento y motivación como predictores del bienestar subjetivo y psicológico
}

\author{
Pilar Sanjuán y María Ávila \\ Universidad Nacional de Educación a Distancia, Madrid, España
}

\begin{abstract}
Resumen: Existe consenso en considerar que la salud mental no consiste simplemente en la ausencia de síntomas psicopatológicos, sino que requiere un estado de bienestar. Por ello, la promoción del bienestar es actualmente una prioridad sanitaria. El principal objetivo de este estudio fue analizar la aportación conjunta que motivación y afrontamiento tienen en el desarrollo de bienestar subjetivo y psicológico. Doscientos participantes (115 varones y 85 mujeres) contestaron a diferentes cuestionarios sobre motivación, afrontamiento y diferentes dimensiones de bienestar subjetivo y psicológico. Los resultados mostraron que motivación y afrontamiento tomados conjuntamente eran predictores significativos de las distintas dimensiones de bienestar analizadas. Se concluye que los programas de promoción del bienestar deben fomentar que las personas persigan sus metas por motivación autónoma, empleen estrategias de afrontamiento basadas en la solución de los problemas, la reestructuración cognitiva positiva y el apoyo social y obvien el uso de las estrategias de evitación.
\end{abstract}

Palabras clave: Bienestar subjetivo; bienestar psicológico; motivación autónoma; estrategias de afrontamiento.

Coping and motivation as predictors of subjective and psychological well-being

\begin{abstract}
Consensus is that mental health is not simply the absence of psychopathological symptoms, but requires a state of well-being. Therefore, the promotion of well-being is now considered a health priority. The main objective of this study was to analyze the joint contribution of motivation and coping to the development of subjective and psychological well-being. Two hundred participants (115 men and 85 women) responded to different questionnaires on motivation, coping and several dimensions of subjective and psychological well-being. The results showed that motivation and coping taken together were significant predictors of the different dimensions of well-being as analyzed. It is concluded that promotion of well-being programs should encourage people to pursue their goals by autonomous motivation, employ coping strategies based on problem solving, positive cognitive restructuring and social support and obviates the use of avoidance strategies.
\end{abstract}

Keywords: Subjective well-being; psychological well-being; autonomous motivation; coping strategies.

\section{Introducción}

En la actualidad existe acuerdo en considerar que la salud mental no es la mera ausencia de síntomas como ansiedad o depresión (Keyes, 2007), y, además, que la experimentación de bienestar puede contribuir a reducir el riesgo para desarrollar una enfermedad mental en el futuro (Quiceno, Mateus, Cárdenas, Villarreal y Vinac-

Recibido: 10 octubre 2015; aceptado: 15 diciembre 2015.

Correspondencia: Pilar Sanjuán, Facultad de Psicología, Universidad Nacional de Educación a Distancia, Juan del Rosal, 10, 28040 Madrid, España. Correo-e: psanjuan@psi.uned.es cia, 2013; Keyes, Dhingra, y Simoes, 2010). Verdaderamente, el bienestar forma parte consustancial de lo que se entiende por salud, y ya la Organización Mundial de la Salud (OMS) en su acta de constitución en 1948, la definió como un estado completo de bienestar físico, mental y social y no meramente la ausencia de enfermedades o trastornos. Hasta hace muy poco tiempo, el bienestar se había quedado fuera de los informes de la organización, que se había centrado en otros objetivos. Sin embargo, en la actualidad este panorama ha cambiado y recientemente la OMS ha considerado que la mejora del bienestar de la población constituye una prioridad sanitaria (World Health Organization: WHO, 2013), pos- 
tura que también queda recogida en la nueva estrategia sanitaria de la Europa de 2020 (Comisión Europea, 2010). El reconocimiento del bienestar como una prioridad sanitaria se debe, en parte, a los esfuerzos que se han realizado, fundamentalmente desde la psicología, para abordar su conceptualización y evaluación desde una perspectiva científica. Por otra parte, la promoción del bienestar requiere que se conozcan todos aquellos procesos, mecanismos o variables que favorecen su desarrollo (Martxueta y Exebarria, 2014), por lo que seguir investigando sobre estos aspectos sigue siendo necesario.

El bienestar es un constructo complejo y se han ido desarrollado diferentes conceptualizaciones que resaltan distintos aspectos de lo que constituye estar bien. De acuerdo con Ryan y Deci (2001), existen dos grandes aproximaciones en la conceptualización del bienestar, la hedónica y la eudaimónica, que se centran en sentirse bien y funcionar bien, respectivamente. Tal y como se va a exponer a continuación, ambas aproximaciones se complementan, por lo que un abordaje completo del bienestar requiere que las dos sean tenidas en cuenta.

Dentro de la perspectiva centrada en sentirse bien (o perspectiva hedónica), la aproximación empírica más importante es la denominada como bienestar subjetivo, puesto que resalta que la percepción subjetiva que las personas tienen de sus vidas es mucho más relevante que los índices objetivos sobre la calidad de vida. En cualquier caso, es necesario aclarar que, aunque el bienestar sea un juicio subjetivo, no implica que no pueda ser estudiado empíricamente. Por otra parte, el hecho de que sea un juicio subjetivo tampoco significa que no se relacione con variables objetivas. De hecho, las personas que se sienten felices, también son evaluadas como felices por otras personas, recuerdan más eventos positivos, sonríen más y presentan respuestas fisiológicas acordes con el sentimiento de felicidad (Lyubomirsky, Sheldon y Schkade, 2005). El bienestar subjetivo se define como la evaluación que hace la persona de su vida. Esta evaluación incluye tanto juicios cognitivos sobre la satisfacción vital, como valoraciones afectivas sobre sus estados de ánimo y emociones. Por lo tanto, se puede decir que el bienestar subjetivo tiene dos componentes, uno afectivo y otro cognitivo. En relación con el componente afectivo, las personas con bienestar subjetivo informarían de experiencia frecuente de emociones positivas y la baja proporción de emociones negativas, $\mathrm{y}$ con respecto al componente cognitivo, juzgarían que están satisfechas con su vida (Diener, Lucas y Oishi, 2002).

Desde la perspectiva focalizada en funcionar bien (o perspectiva eudaimónica), se resalta que aunque las personas informen sentirse bien o felices, eso no significa que funcionen bien psicológicamente. Se considera que este funcionamiento psicológico óptimo se logra cuando la persona desarrolla su verdadero potencial, o lo que es lo mismo, cuando existe una congruencia entre sus objetivos y su self. Se podría decir que los psicólogos de esta perspectiva han recogido el legado de la psicología humanista, y han basado sus propuestas teóricas en los conceptos desarrollados por Allport, Maslow o Rogers. Pero además, han dado un paso más allá, y han llevado a cabo muchos estudios empíricos que fundamentan estas propuestas teóricas (Ryff y Singer, 2008).

En esta línea, una de las propuestas más integradoras es el modelo de bienestar psicológico propuesto por Ryff (1989a). Esta autora basó su formulación no sólo en la psicología humanista, sino también en la psicología del desarrollo, así como en otros criterios de salud mental de la psicología clínica. Ryff (1989a,b) considera que el bienestar psicológico es un constructo multidimensional que incluye diferentes actitudes vitales tales como: 1) autoaceptación, que implica que la persona llegue a conocer y aceptar tanto sus fortalezas como sus limitaciones; 2) experimentar relaciones positivas con los otros, que implica que las personas desarrollen y mantengan buenas relaciones interpersonales; 3 ) autonomía, que se refiere al desarrollo de un sentido de autodeterminación, independencia y regulación de la conducta; 4) dominio del entorno, que significa que la persona pueda escoger, adaptar o crear ambientes que puedan satisfacer sus necesidades personales; 5) propósito vital, que hace referencia a que la persona esté implicada en el logro de metas significativas, y 6) crecimiento personal, que implica el proceso continuo de desarrollar los propios potenciales.

Algunos de los factores que tienen un potente efecto sobre el desarrollo de bienestar, y que han sido destacados por diferentes autores (Diener, Suh, Lucas, y Smith, 1999; Lyubomirsky, King, y Diener, 2005; Ryff, 2014), son la implicación en metas y el uso de un afrontamiento efectivo.

En relación con las metas, diferentes estudios han mostrado que el progreso y/o logro de las metas se asocia con el bienestar, tanto en muestras de estudiantes como de adultos, y en distintos dominios como el académico, el deportivo o las relaciones de pareja (Amiot, Gaudreau, y Blanchard, 2004; Hortop, Wrosch, y Gagne, 2013; Sheldon y Elliot, 1999; Smith, Ntoumanis, y Duda, 2007; Smith, Ntoumanis, Duda, y Vansteenkiste, 2011; Wiese, 2007). Dentro de esta línea de estudio, Sheldon y Elliot (1999) propusieron que el bienestar experimentado por el logro de las metas, se derivaría en gran medida de la motivación que guía la persecución de dichas metas. 
De acuerdo con la Teoría de la Auto-Determinación (Ryan y Deci, 2008), los motivos por los que se persiguen las metas pueden ser clasificados en: (1) autónomos o auto-determinados (i.e., basados en el interés personal, el disfrute o la percepción de importancia), y (2) controlados o no-auto-determinados (i.e., derivados de presiones internas o externas, o contingencias relacionadas con la aprobación social). En concreto, los motivos autónomos incluyen la persecución de la meta porque ésta refleja los valores propios (motivos integrados) y/o por su importancia inherente (motivos identificados). Por otra parte, los motivos controlados incluyen perseguir la meta por sentimiento de culpa u otras presiones internas (motivos introyectados) y/o porque alguien quiere o porque existen ganancias o pérdidas específicas (por ej., dinero) asociadas con el resultado (motivos externos).

Lo que Sheldon y Elliot (1999) sugirieron, es que sólo el logro de las metas guiadas por motivos autónomos se relaciona con bienestar. Los resultados de algunos estudios posteriores han apoyado esta propuesta, pues han encontrado que los motivos autónomos mantienen una relación positiva con el bienestar subjetivo (Hortop et al., 2013; Miquelon y Vallerand, 2006; Sheldon y Elliot, 1999; Sheldon, Ryan, Deci, y Kasser, 2004; Smith et al., 2011), mientras que los motivos controlados muestran una relación inversa (Amiot et al., 2004; Hortop et al, 2013; Miquelon y Vallerand, 2006).

El afrontamiento, otro de los factores con mayor impacto en el bienestar, se define como las acciones tanto conductuales como cognitivas que las personas emplean en respuesta a las demandas externas e internas que consideran que exceden a sus recursos (Lazarus y Folkman, 1984). Tradicionalmente, las diferentes estrategias de afrontamiento se han conceptualizado como centradas en la solución del problema o en el manejo de la respuesta emocional que se deriva del mismo (Lazarus y Folkman, 1984). Sin embargo, hay que tener en cuenta que esta clasificación no es totalmente excluyente, ya que las estrategias centradas en el problema pueden conseguir solucionarlo o no, lo que, en cualquier caso, derivará en una respuesta emocional del individuo. El propio Lazarus (2006) ha señalado en un escrito posterior, que el afrontamiento siempre se refiere a los esfuerzos por manejar las demandas adaptativas y las emociones que se generan, y por lo tanto, el afrontamiento se puede considerar como una característica integral del proceso emocional. Investigaciones recientes avalan esta postura pues han encontrado que las estrategias de afrontamiento no tienen un impacto diferencial en los problemas y las emociones (Litman y Lunsford, 2009). Además, los estudios factoriales tampoco encuentran que las diferentes estrategias saturen de forma clara en factores centrados en los pro- blemas o en la emoción (Carver, Scheier y Weintraub, 1989; Litman y Lunsford, 2009; Yi-Frazier et al., 2010).

Por todo ello, en la actualidad es habitual clasificar las distintas estrategias en función de su capacidad para reducir el malestar y/o incrementar el bienestar. En este sentido, la investigación ha encontrado que aquellas estrategias centradas en el control primario y secundario se asocian sistemáticamente con mayor bienestar emocional (Folkman, 2008; Sanjuán y Magallares, 2014). El control primario implica cambiar la situación, es decir, resolver el problema, e incluye tanto las estrategias orientadas a la acción (afrontamiento activo) como las de toma de decisiones (planificación). El control secundario no implica cambiar la situación, sino cambiar uno mismo, es decir, que la persona modifique la percepción que tiene de la situación, o lo que es lo mismo, que realice una reestructuración cognitiva positiva de la misma (por ej., reevaluación positiva o aceptación).

Por otra parte, las estrategias focalizadas en la evitación de la situación se han relacionado de forma consistente con el malestar emocional (Litman y Lunsford, 2009; Sanjuán y Magallares, 2015). Respecto a las estrategias de afrontamiento basadas en la búsqueda de apoyo social, aunque se han asociado con bienestar (Greenglass y Fiksenbaum, 2009), las relaciones no son tan consistentes. Esto puede ser debido a que, mientras que el apoyo instrumental puede contribuir a la solución del problema mediante la ayuda directa o el consejo, el apoyo emocional puede convertirse en una mera descarga de emociones, lo que le colocaría dentro de las estrategias basadas en la evitación (Carver et al., 1989).

La categorización de las distintas estrategias de afrontamiento que emplean las personas sigue siendo un debate abierto entre los expertos en el tema. Sin embargo, algunas categorías, que coinciden con las que hemos descrito en los párrafos anteriores (solución de problemas, reestructuración cognitiva positiva, evitación y apoyo social), aunque denominadas de forma diferente en los distintos modelos, han sido propuestas de forma sistemática en todos ellos (Carver et al., 1989; Lazarus y Folkman, 1984; Skinner, Edge, Altman y Sherwood, 2003). Por otra parte, tampoco existe un total acuerdo en cómo agrupar las categorías, pues mientras que algunos autores consideran que las estrategias focalizadas en el control primario y en el secundario, es decir en la solución de problemas y la reestructuración cognitiva positiva, son categorías separadas (Skinner et al, 2003), otros han encontrado que éstas cargan en un único factor en los análisis factoriales (Litman y Lunsford, 2009; Yi-Frazier et al., 2010).

Con el presente estudio se pretende aportar nuevas evidencias en relación a varios aspectos que quedan por 
aclarar sobre la problemática planteada. En primer lugar, como acabamos de exponer, los estudios que han analizado las relaciones entre afrontamiento y bienestar se han centrado casi exclusivamente en el componente emocional del bienestar subjetivo (Folkman, 2008; Litman y Lunsford, 2009; Sanjuán y Magallares, 2014), por lo que poco se sabe de la relación que las distintas estrategias de afrontamiento mantienen con la satisfacción vital y con los distintos componentes del bienestar psicológico.

En segundo lugar, y tal como se ha señalado, la literatura previa ha mostrado que los motivos autónomos se asocian con el bienestar subjetivo. Sin embargo, la investigación que se ha realizado hasta la fecha, o no ha medido la satisfacción vital (Amiot et al., 2004; Hortop et al., 2013; Miquelon y Vallerand, 2006), o ésta ha sido incluida en un medida promediada con el componente afectivo (Sheldon y Elliot, 1999; Sheldon et al., 2004; Smith et al., 2011). Aunque los componentes del bienestar subjetivo están relacionados, son diferentes y los expertos defienden el empleo de medidas separadas de éstos, ya que muestran un patrón asociativo distinto con las variables analizadas (Schimmack, Schupp, y Wagner, 2008).

En tercer lugar, los estudios realizados hasta la fecha se han focalizado en la relación que los motivos o el afrontamiento, de forma independiente, tienen con el bienestar, pero no se ha estudiado su contribución conjunta al mismo. Por todo ello, el principal objetivo del presente estudio es analizar la contribución conjunta que los motivos y el afrontamiento tienen en cada una de las distintas dimensiones del bienestar subjetivo y psicológico tomadas por separado.

De acuerdo con las evidencias y propuestas presentadas previamente, esperamos que los motivos autónomos, las estrategias centradas en la solución de problemas, la reestructuración cognitiva positiva y la búsqueda de apoyo social sean predictores positivos de los distintos componentes del bienestar subjetivo (balance afectivo y satisfacción vital) y psicológico (autoaceptación, propósito vital y crecimiento personal), mientras que las estrategias de evitación sean predictores negativos de los mismos.

\section{Método}

\section{Participantes}

Doscientas personas (115 varones y 85 mujeres) con edades comprendidas entre los 20 y los 56 años (media $=36.79$ y $D T=9.61$ ) participaron voluntariamente en el estudio. La mayoría trabajaba (75.5\%) y vivía acompañada de su familia (83\%) en el momento de la recogida de los datos. El 13.5\% tenía estudios primarios, el 38\% secundarios y el $48.5 \%$ tenía estudios universitarios.

\section{Medidas y procedimiento}

A todas las personas que estuvieron de acuerdo en participar en el estudio se les pidió que completaran los siguientes cuestionarios:

Cuestionario de Motivos. Ha sido desarrollado por Sheldon y su equipo (Sheldon y Elliot, 1999; Sheldon et al., 2004), obteniendo coeficientes alpha comprendidos entre .60 y .90 . El cuestionario ha sido traducido al español para los propósitos de este estudio. A los participantes se les pedía que expusieran tres metas personales que estuvieran intentando lograr en el período de tiempo en el que se realizó la evaluación. A través de 12 ítems con escalas tipo Likert de 7 puntos, los participantes valoraban el grado en el que perseguían cada una de estas 3 metas por motivos externos ("porque alguien quiere que lo haga o la situación lo requiere"), introyectados ("para evitar sentir vergüenza, culpa o ansiedad"), identificados ("por su importancia") o intrínsecos ("por la satisfacción que produce"), siendo el "0" equivalente a "Nada" y el "6" a "Totalmente". Siguiendo el procedimiento propuesto por Sheldon y colaboradores (Sheldon y Elliot, 1999; Sheldon et al., 2004), se calcularon primero dos puntuaciones, una equivalente a los motivos controlados, haciendo la media de las puntuaciones dadas a los ítems que evaluaban los motivos externos e introyectados; y otra equivalente a los motivos autónomos, haciendo la media de las puntuaciones dadas a los ítems que valoraban los motivos identificados e intrínsecos. Después, se calculó una puntuación denominada "autonomía relativa de los motivos" (ARM), restando los motivos controlados de los autónomos. Cuanto más elevada es esta puntuación, mayor prevalencia de los motivos autónomos en la persecución de las metas. Los coeficientes alpha en la presente muestra fueron .73 para los motivos controlados y .53 para los autónomos.

Cuestionario de Afrontamiento COPE abreviado. La versión original fue desarrollada por Carver (1997) que ha informado coeficientes alpha que oscilaban entre .50 y .90 en función de la subescala. La traducción y adaptación al español ha sido llevada a cabo por Morán, Landero y González (2010). El cuestionario consta de 28 ítems que valoran 14 estrategias de afrontamiento diferentes. Los participantes señalan mediante escalas tipo Likert de 7 puntos el grado con que emplean cada una de las estrategias recogidas, siendo el " 0 " "Nada en absoluto" y el "6" "Totalmente". De acuerdo con la literatura sobe afrontamiento (Skinner et al., 2003), y la recomendación del propio Carver (1997), las diferentes estrategias se agruparon en cuatro categorías más amplias: afrontamiento basado en la solución de los problemas (4 ítems que valoran estrategias de afrontamiento activo y plani- 
ficación), afrontamiento basado en la reestructuración cognitiva positiva (4 ítems que recogen estrategias de reevaluación positiva de la situación y aceptación), afrontamiento basado en la búsqueda de apoyo social (4 ítems que se refieren a las estrategias de apoyo emocional e instrumental) y afrontamiento basado en la evitación (10 ítems que evalúan estrategias de negación, desimplicación, uso de sustancias, desahogo y autoculpa). Se calcularon 4 puntuaciones haciendo la media de las puntuaciones dadas a los ítems que se incluían en cada una de las 4 categorías. Puntuaciones elevadas indican mayor uso de cada una de estas estrategias. En la muestra presente, los coeficientes alpha alcanzados fueron de .69 para el afrontamiento de solución de problemas, .61 para el basado en la reestructuración cognitiva positiva, .86 para el basado en la búsqueda de apoyo social y .74 para el basado en la evitación.

Escalas de Afecto Positivo y Negativo (PANAS). Esta escala fue originalmente desarrollada por Watson, Clark, y Tellegen (1988), que informaron coeficientes de fiabilidad entre .86 y .90 para afecto positivo y entre .84 a .87 para afecto negativo. En este estudio hemos empleado la adaptación al español llevada a cabo por Sandín et al. (1999). La escala consta de 20 ítems que se corresponden con 20 adjetivos que recogen diferentes emociones, 10 positivas y 10 negativas. Se pedía a los participantes que respondieran el grado en que sentían cada una de las emociones de forma habitual empleando escalas de 7 puntos que iban de "0" ("Nada, en absoluto") a "6" ("Muchísimo"). Se calcularon dos puntuaciones correspondientes al afecto positivo y negativo haciendo la media de las puntuaciones dadas a cada uno de los ítems que componen cada una de estas dos subescalas. Posteriormente, el afecto negativo se restó del positivo para calcular el balance afectivo. De esta manera, una puntuación positiva indicaría predominancia del afecto positivo sobre el negativo, mientras que una puntuación negativa señalaría una predominancia del afecto negativo sobre el positivo. Una ventaja del cálculo del balance afectivo sobre las medidas unidimensionales de afecto positivo y negativo, es que permite el control de los sesgos derivados de puntuaciones muy extremas (Schimmack y Diener, 1997). En la muestra actual, los coeficientes alpha para las subescalas de afecto positivo y negativo fueron de .80 y .86 respectivamente.

Escala de Satisfacción con la Vida. Ha sido desarrollada originalmente por Pavot y Diener (1993), obteniendo un coeficiente alpha de .87. La adaptación española empleada en el presente estudio fue realizada por Vázquez, Duque, y Hervás (2012). Consiste en 5 ítems que miden la satisfacción con la vida de forma global en lugar de en dominios específicos. Los participantes valo- ran el grado de acuerdo con los ítems mediante escalas tipo Likert de 7 puntos, donde el " 0 " significa "Totalmente en desacuerdo" y el "6" "Totalmente de acuerdo". Se calcula una puntuación haciendo la media de las puntuaciones dadas a cada uno de los 5 ítems que componen la escala. Las puntuaciones elevadas indican mayor satisfacción con la vida. En el presente estudio el coeficiente alpha alcanzado por la escala fue de .85 .

Escalas de Bienestar Psicológico. Fue desarrollada por Ryff (1989), que informó de coeficientes de consistencia interna que oscilaban entre .70 y .84 en función de la subescala considerada. La adaptación española empleada en el presente estudio fue realizada por Diaz y colaboradores (2006). La escala completa consta de 29 ítems que valoran, de acuerdo con Ryff, las 6 dimensiones que se corresponden con un funcionamiento psicológico óptimo. Para los propósitos de este estudio, sólo se han empleado 3 de estas subescalas: la autoaceptación (4 ítems), el propósito vital (5 ítems), y el crecimiento personal (4 ítems). Cada uno de los ítems se puntúa con una escala tipo Likert de 7 puntos desde el " 0 " ("Totalmente en desacuerdo") a "6" ("Totalmente de acuerdo"). Las tres puntuaciones se calcularon haciendo la media de las puntuaciones dadas a cada uno de los ítems que componen las subescalas. Las puntuaciones altas en estas subescalas indican mayor autoaceptación, propósito vital y crecimiento personal, respectivamente. Los coeficientes alpha alcanzados fueron de .78 para autoaceptación, .84 para propósito vital y .56 para crecimiento personal.

\section{Resultados}

Con el fin de comprobar si las variables sociodemográficas (edad, sexo, nivel de estudios, estatus como trabajador y tipo de convivencia) mantenían alguna relación con las variables psicológicas de interés, se calcularon correlaciones de Pearson entre ellas. De esta manera, se comprobó que la edad mantenía correlaciones negativas estadísticamente significativas con balance afectivo $(-.15, p<.05)$, satisfacción vital $(-.20, p<.005)$, autoaceptación $(-.14, p<.05)$, propósito vital $(-.15, p<.05) \mathrm{y}$ crecimiento personal $(-.14, p<.05)$, lo que implica que cuanta mayor edad, menos bienestar. Por otra parte, el nivel de estudios mostró una correlación negativa significativa con propósito vital $(-.19, p<.01)$, lo que indica que cuanto más elevado es el nivel de estudios, menor es el propósito vital informado. Por último, el sexo mantenía una correlación marginalmente significativa con el crecimiento personal $(.13, p=.06)$, en el sentido de que las mujeres parecían experimentar mayor crecimiento personal. Con el objetivo de controlar su efecto, la edad, 
el nivel de estudios y el sexo se incluyeron en los análisis posteriores cuando se estudiaron las variables psicológicas con las que mantenían una relación.

Como paso previo a los análisis de interés, procedimos a calcular las correlaciones de Pearson que los motivos y el afrontamiento mantenían con las distintas dimensiones del bienestar. Tal y como podemos ver en la Tabla 1, motivos y afrontamiento correlacionaron con todas las medidas de bienestar salvo con las estrategias basadas en la reestructuración cognitiva y en la evitación, que no mantuvieron relaciones con la satisfacción vital, y el afrontamiento basado en la búsqueda de apoyo social, que no correlacionó con balance afectivo. De acuerdo con el signo de las correlaciones, se puede decir que se informa más bienestar cuanto más autónomos son los motivos, más se emplea el afrontamiento de solución de problemas, de reestructuración cognitiva positiva y de apoyo social, y menos se usa el de evitación.

Tabla 1. Correlaciones de motivos y afrontamiento con medidas de bienestar subjetivo y psicológico

\begin{tabular}{llcccc}
\hline & ARM & Afr. SP & Afr. RCP & Afr. Apoyo & Afr. Evitación \\
\hline Balance afectivo & $.28^{* * *}$ & $.29 * * *$ & $.30 * * *$ & .07 & $-.52 * * *$ \\
Satisfacción vital & $.23 * * *$ & $.24 * * *$ & .07 & $.24 * * *$ & -.11 \\
Autoaceptación & $.20^{* *}$ & $.43 * * *$ & $.28^{* * *}$ & $.23^{* * *}$ & $-.26^{* * *}$ \\
Propósito vital & $.21 * *$ & $.49 * * *$ & $.26^{* * *}$ & $.28^{* * *}$ & $-.21^{* *}$ \\
Crecimiento personal & $.32 * * *$ & $.38^{* * *}$ & $.24 * *$ & $-.18^{* *}$ \\
\hline
\end{tabular}

Nota $:$ ARM = Autonomía Relativa de los Motivos; Afr. SP = Afrontamiento basado en la Solución de Problemas; Afr. RCP = Afrontamiento basado en la Reestructuración Cognitiva Positiva. ${ }^{* *} p<.01 ; * * p<.001$

Para estudiar la contribución conjunta que la motivación y el afrontamiento pueden tener en los distintos componentes del bienestar subjetivo y psicológico, procedimos a realizar diferentes análisis de regresión, uno por cada componente del bienestar, introduciendo como predictores las distintas estrategias de afrontamiento y la autonomía relativa de los motivos. En la Tabla 2 pueden verse los resultados obtenidos en estos análisis.

Como podemos ver en la Tabla 2, los predictores analizados explicaron en conjunto un $35 \%$ de la varianza del balance afectivo. La edad, que fue incluida para poder controlar su posible efecto en la variable criterio, perdió su significación al tener en cuenta todos los predictores conjuntamente. De acuerdo con las significaciones encontradas, se podría decir que cuanto más se emplean las estrategias de solución de problemas y de reestructuración cognitiva positiva, y menos las de evitación, mayor balance afectivo se muestra.

De acuerdo con los resultados obtenidos, todos los predictores explicaron conjuntamente el $17 \%$ de la varianza de la satisfacción vital. En este caso, los datos muestran que, después de controlar el efecto de la edad, cuanto más autónomos son los motivos y más se emplean las estrategias de solución de problemas y de apoyo social, se informa de más satisfacción.

El conjunto de todos los predictores analizados explicaron el $28 \%$ de la varianza de la autoaceptación. Teniendo en cuenta las significaciones, y después de controlar el efecto de la edad, se podría decir que cuanto más se emplean las estrategias de solución de problemas y de apoyo social y menos las de evitación, se informa de más autoaceptación.

Todos los predictores incluidos explicaron un 36\% de la varianza de las puntuaciones del propósito vital. Si nos detenemos en las significaciones, y después de controlar el efecto de la edad y del nivel de estudios, se puede decir que se informa de más propósito vital cuanto más autónomos son los motivos que guían las metas, más se emplean las estrategias de solución de problemas y de apoyo social, y menos se utilizan las estrategias basadas en la evitación.

Todos los predictores conjuntamente explicaron el $27 \%$ de la varianza de las puntuaciones en crecimiento personal. Los datos muestran que, después de controlar el efecto de la edad y el sexo, cuanto más autónomos son los motivos subyacentes y más se emplea el afrontamiento basado en la solución de los problemas, se informa de mayor crecimiento personal.

\section{Discusión}

El objetivo principal de este estudio fue analizar la contribución conjunta que la motivación y el afrontamiento tienen en los distintos componentes del bienestar subjetivo y psicológico. En general, los resultados apoyan que la motivación y el afrontamiento tienen un peso importante en el bienestar, explicando una parte significativa de su varianza, concretamente, entre el 17 y el $36 \%$, dependiendo de la dimensión del bienestar de la que se trate. El conjunto de resultados obtenidos nos permiten sugerir que para que las personas puedan sentirse bien y funcio- 
Tabla 2. Análisis de regresión para predecir los distintos componentes del bienestar subjetivo y psicológico

\begin{tabular}{|c|c|c|c|c|c|c|}
\hline \multirow[b]{2}{*}{ Criterio } & \multirow[b]{2}{*}{ Predictores } & \multicolumn{3}{|c|}{ Modelo } & \multirow[b]{2}{*}{$\beta$} & \multirow[b]{2}{*}{$t$} \\
\hline & & $R^{2}$ & $F$ & g.l. & & \\
\hline \multirow[t]{7}{*}{ Balance afectivo } & & .35 & $16.66^{* * *}$ & 6,193 & & \\
\hline & Edad & & & & -.02 & -0.41 \\
\hline & ARM & & & & .10 & 1.57 \\
\hline & Afr. SP & & & & .18 & $2.94 * *$ \\
\hline & Afr. RCP & & & & .13 & $2.11 *$ \\
\hline & Afr. Apoyo Social & & & & .05 & 0.83 \\
\hline & Afr. Evitación & & & & -.44 & $-6.68 * * *$ \\
\hline \multirow[t]{7}{*}{ Satisfacción vital } & & .17 & $6.46^{* * *}$ & 6,193 & & \\
\hline & Edad & & & & -.14 & $1.99 *$ \\
\hline & ARM & & & & .18 & $2.46 * *$ \\
\hline & Afr. SP & & & & .22 & $3.09 * * *$ \\
\hline & Afr. RCP & & & & -.06 & -0.87 \\
\hline & Afr. Apoyo Social & & & & .19 & $2.78 * *$ \\
\hline & Afr. Evitación & & & & -.03 & -0.42 \\
\hline \multirow[t]{7}{*}{ Autoaceptación } & & .28 & $12.78 * * *$ & 6,193 & & \\
\hline & Edad & & & & -.06 & -0.99 \\
\hline & ARM & & & & .10 & 1.53 \\
\hline & Afr. SP & & & & .34 & $5.27 * * *$ \\
\hline & Afr. RCP & & & & .10 & 1.55 \\
\hline & Afr. Apoyo Social & & & & .15 & $2.35 * *$ \\
\hline & Afr. Evitación & & & & -.17 & $-2.43 * *$ \\
\hline \multirow[t]{8}{*}{ Propósito vital } & & .36 & $15.22 * * *$ & 7,192 & & \\
\hline & Edad & & & & -.07 & -1.19 \\
\hline & Nivel de estudios & & & & -.15 & $-2.65 * *$ \\
\hline & ARM & & & & .13 & $2.05^{*}$ \\
\hline & Afr. SP & & & & .40 & $6.36 * * *$ \\
\hline & Afr. RCP & & & & .06 & 0.96 \\
\hline & Afr. Apoyo Social & & & & .20 & $3.22 * * *$ \\
\hline & Afr. Evitación & & & & -.12 & $-1.77^{\circ}$ \\
\hline \multirow[t]{8}{*}{ Crecimiento personal } & & .27 & $9.932 * * *$ & 7,192 & & \\
\hline & Edad & & & & -.07 & -1.03 \\
\hline & Sexo & & & & .06 & 1.03 \\
\hline & ARM & & & & .27 & $3.99 * * *$ \\
\hline & Afr. SP & & & & .31 & $4.69 * * *$ \\
\hline & Afr. RCP & & & & .10 & 1.51 \\
\hline & Afr. Apoyo Social & & & & .08 & 1.20 \\
\hline & Afr. Evitación & & & & -.02 & -0.26 \\
\hline
\end{tabular}

Nota $:$ ARM = Autonomía Relativa de los Motivos; Afr. SP = Afrontamiento basado en la Solución de Problemas; Afr. RCP = Afrontamiento basado en la Reestructuración Cognitiva Positiva. Nivel de estudios: $1=$ Primarios, $2=$ Secundarios y $3=$ Universitarios. Sexo: 1 = varón y 2 mujer. ${ }^{\circ} p=.08 ; * p<.05 ; * * p<.01 ; * * * p<.001$

nar de forma adecuada psicológicamente, es importante que persigan metas que consideren importantes y que lo hagan por motivos autónomos, es decir, por la gratificación inherente a las mismas. También que en este proceso empleen estrategias de afrontamiento efectivas, basadas en la solución de los problemas, en la reevaluación positiva de las situaciones y el apoyo social, a la vez que obvien las estrategias basadas en la evitación como la negación, la desimplicación o el uso de sustancias.
Los motivos, cuando fueron estudiados de forma aislada, se asociaron con todas las dimensiones tanto del bienestar subjetivo como del psicológico, indicando que cuanto más autónomos son los motivos, más bienestar se informa. Estos resultados corroboran los obtenidos en otros estudios previos con respecto a la asociación entre la autonomía de los motivos y el componente afectivo del bienestar subjetivo (Amiot et al., 2004; Hortop et al., 2013; Miquelon y Vallerand, 2006), y aportan nueva evi- 
dencia sobre las relaciones mantenidas con los otros componentes del bienestar. Sin embargo, cuando los motivos se analizaron conjuntamente con las diferentes estrategias de afrontamiento, perdieron su capacidad para predecir el balance afectivo y la autoaceptación. En el caso de la satisfacción vital, el propósito vital y el crecimiento personal, tanto motivos como afrontamiento resultaron predictores significativos cuando se estudiaron sus aportaciones de forma conjunta.

De la misma manera, algunas estrategias de afrontamiento concretas que se asociaban significativamente con las distintas dimensiones del bienestar cuando se estudiaron independientemente, perdieron su significación al analizarse de forma conjunta. Así, las estrategias basadas en la reestructuración cognitiva positiva dejaron de ser un predictor significativo para las tres dimensiones del bienestar psicológico analizadas, autoaceptación, propósito vital y crecimiento personal. En la misma línea, las estrategias basadas en la búsqueda de apoyo social y las de evitación dejaron de ser predictores significativos del crecimiento personal, aunque de forma independiente sí habían mostrado asociaciones. Por el contrario, hay que señalar que el empleo de estrategias basadas en la solución de los problemas se ha mantenido como un predictor significativo para todas las dimensiones de bienestar cuando se ha analizado la contribución conjunta de los diversos predictores.

De esta manera, nuestros resultados, además de apoyar aquellos encontrados cuando se han analizado los componentes emocionales del bienestar (Folkman, 2008; Litman y Lunsford, 2009; Sanjuán y Magallares, 2014), ponen de manifiesto que las estrategias de solución de problemas son también fundamentales para facilitar el desarrollo de otros componentes del bienestar de naturaleza más cognitiva. Los datos también muestran que la estrategia de búsqueda de apoyo social es un predictor positivo para algunas de estas dimensiones cognitivas del bienestar, como la satisfacción vital, la autoaceptación y el propósito vital, mientras que no resulta relevante para el balance afectivo. Nuestros datos corroboran que el empleo de estrategias de evitación es un predictor negativo de bienestar emocional, tal y como otros estudios habían mostrado previamente (Litman y Lunsford, 2009; Sanjuán y Magallares, 2015). Además, ponen de manifiesto que también suponen un predictor negativo para componentes como la autoaceptación y el propósito vital.

El hecho de que no todos los predictores sean significativos en todas las dimensiones del bienestar estudiadas apoya la idea de que es necesario analizar los diferentes componentes del bienestar por separado, tal como otros autores han propuesto (Schimmack et al., 2008), de cara a conocer de forma precisa qué procesos se deben fo- mentar para promover el desarrollo de cada una de las diferentes dimensiones del bienestar.

En futuras investigaciones, sería interesante incorporar el estudio de otros factores que se sabe que tienen un rol en el desarrollo del bienestar, tales como la perspectiva optimista, el propio estado de salud física del individuo, o la percepción que la persona tiene del mismo (Deneve y Cooper, 1998; Sanjuán, 2014), de tal manera que pudiésemos conocer las distintas aportaciones que cada uno de los factores tiene cuando actúan conjuntamente, incrementando así la validez ecológica.

Nuestro estudio muestra algunas limitaciones que es preciso mencionar. Por una parte, al emplearse un diseño transversal, no podemos establecer de forma precisa la dirección de las relaciones que motivación y afrontamiento mantienen con el desarrollo de las distintas dimensiones del bienestar. En futuros estudios sobre estos temas sería recomendable incorporar una perspectiva longitudinal que nos permitiera establecer claramente cómo motivos y afrontamiento afectan al desarrollo de las distintas dimensiones del bienestar.

Por otra parte, en el estudio no se ha evaluado el progreso hacia las metas de los participantes, lo que también es una limitación. Aunque los resultados obtenidos parecen sugerir que la relación positiva entre motivos y bienestar es independiente del progreso y/o logro de las metas, sería necesario comprobarlo empíricamente en estudios posteriores. Por otra parte, un diseño longitudinal, además de permitirnos probar las relaciones causales entre motivos, afrontamiento y bienestar, tal como se ha señalado previamente, también nos ayudaría a estudiar estas asociaciones a través de todo el proceso de logro de las metas y comprobar el rol jugado por el progreso hacia las mismas.

No obstante, a pesar de las limitaciones señaladas, el presente estudio aporta evidencia sobre los factores que tienen un impacto en el bienestar, lo que resulta esencial para planificar programas de promoción basados en la evidencia. Aunque es necesario seguir realizando estudios, tal y como hemos apuntado, los resultados obtenidos aquí sugieren que estos programas deben dirigirse a fomentar que las personas persigan sus metas por motivos autónomos, que empleen estrategias efectivas como la solución de problemas, la reevaluación positiva de las situaciones o el apoyo social, a la vez que rechacen el uso de las estrategias basada en la evitación como la negación, la desimplicación o el uso de sustancias.

\section{Referencias}

Amiot, C., Gaudreau, P., \& Blanchard, C. (2004). Self-Determination, coping, and goal attainment in sport. Journal of Sport and Exercise Psychology, 26, 396-411. 
Carver, C. (1997). You want to measure coping but your protocol's too long: Consider the Brief COPE. International Journal of Behavioral Medicine, 4, 92-100. http://dx.doi.org/10.1207/ s15327558ijbm0401_6

Carver, C. S., Scheier, M. F., \& Weintraub, J. K. (1989). Assessing coping strategies: A theoretically based approach. Journal of Personality and Social Psychology, 56, 267-283. http://dx.doi. org/10.1037/0022-3514.56.2.267

Deneve, K., \& Cooper, H. (1998). The happy personality: A meta-analysis of 137 personality traits and subjective well-being. Psychological Bulletin, 124, 197-229. http://dx.doi. org/10.1037/0033-2909.124.2.197

Comisión Europea (2010). Europa 2020: Una estrategia para un crecimiento inteligente, sostenible e integrador. Bruselas: Comisión Europea.

Díaz, D., Rodríguez-Carvajal, R., Blanco, A., Moreno-Jiménez, B., Gallardo, I., Valle, C., y Van Dierendonck, D. (2006). Adaptación española de las escalas de bienestar psicológico de Ryff. Psicothema, 18, 572-577.

Diener, E., Lucas, R., \& Oishi, S. (2002). Subjective well-being: The science of happiness and life satisfaction. En C. Snyder y S. Lopez (Eds.), Handbook of positive psychology (pp. 63-73). New York: Oxford University Press.

Diener, E., Suh, E. M., Lucas, R. E., \& Smith, H. L. (1999). Subjective well-being: Three decades of progress. Psychological Bulletin, 125, 276-302. http://dx.doi.org/10.1037/00332909.125.2.276

Folkman, S. (2008). The case for positive emotions in the stress process. Anxiety, Stress, and Coping, 21, 3-14. http://dx.doi. org/10.1080/10615800701740457

Greenglass, E. R., \& Fiksenbaum, L. (2009). Proactive coping, positive affect, and well-being: Testing for mediation using path analysis considerations. European Psychologist, 14, 2939. http://dx.doi.org/10.1027/1016-9040.14.1.29

Hortop, E., Wrosch, C., \& Gagne, M. (2013). The why and how of goal pursuits: Effects of global autonomous motivation and perceived control on emotional well-being. Motivation and Emotion, 37, 675-687. http://dx.doi.org/10.1007/s11031-0139349-2

Keyes, C. (2007). Promoting and protecting mental health as flourishing: A complementary strategy for improving national mental health. American Psychologist, 62, 95-108. http://dx. doi.org/10.1037/0003-066X.62.2.95

Keyes, C., Dhingra, S., \& Simoes, E. (2010). Change in level of positive mental health as a predictor of future risk of mental health. American Journal of Public Health, 100, 2366-2371. http://dx.doi.org/10.2105/AJPH.2010.192245

Lazarus, R. (2006). Emotions and interpersonal relationships: Toward a person-centered conceptualization of emotions and coping. Journal of Personality, 74, 9-46. http://dx.doi.or$\mathrm{g} / 10.1111 / \mathrm{j} .1467-6494.2005 .00368 . \mathrm{x}$

Lazarus, R., \& Folkman, S. (1984). Stress, appraisal, and coping. New York: Springer. [Traducción española de 1986 como Estrés y procesos cognitivos. Barcelona: Martínez Roca].

Litman, J., \& Lunsford, G. (2009). Frequency of use and impact of coping strategies assessed by the COPE Inventory and their relationships to post-event health and well-being. Journal of Health Psychology, 14, 982-991. http://dx.doi. org/10.1177/1359105309341207
Lyubomirsky, S., King, L., \& Diener, E. (2005). The benefits of frequent positive affect: Does happiness lead to success? Psychological Bulletin, 131, 803-855. http://dx.doi. org/10.1037/0033-2909.131.6.803

Lyubomirsky, S., Sheldon, K., \& Schkade, D. (2005). Pursuing happiness: The architecture of sustainable change. Review of General Psychology, 9, 111-131. http://dx.doi. org/10.1037/1089-2680.9.2.111

Martxueta, A., y Etxeberria, J. (2014). Análisis diferencial retrospectivo de las variables de salud mental en lesbianas, gais y bisexuales (LGB) víctimas de bullying homofóbico en la escuela. Revista de Psicopatología y Psicología Clínica, 19, 2335 .

Miquelon, P., \& Vallerand, R. (2006). Goal motives, well-being, and physical health: Happiness and self-realization as psychological resources under challenge. Motivation and Emotion, 30, 259-272. http://dx.doi.org/10.1007/s11031-006-9043-8

Morán, C., Landero, R., \& González, M. (2010). COPE-28: A psychometric analysis of Brief-COPE Spanish version. Universitas Psychologica, 9, 543-552.

Pavot, W., \& Diener, E. (1993). Review of the Satisfaction with Life Scale. Psychological Assessment, 5, 164-172. http://dx. doi.org/10.1037/1040-3590.5.2.164

Quiceno, J. M., Mateus, J., Cardenas, M., Villareal, D., y Vinaccia, S. (2013). Calidad de vida, resiliencia e ideación suicida en adolescentes víctimas de abuso sexual. Revista de Psicopatología y Psicología Clínica, 18, 107-117.

Ryan, R., \& Deci, E. (2001). On happiness and human potentials: A review of research on hedonic and eudaimonic well-being. Annual Review of Psychology, 52, 141-166. http://dx.doi. org/10.1146/annurev.psych.52.1.141

Ryan, R., \& Deci, E. (2008). Self-determination theory and the role of basic psychological needs in personality and the organization of behavior. En O. John, R. Robins y L. Pervin (Eds.), Handbook of personality: Theory and research (pp. 654-678). New York: Guilford Press.

Ryff, C. (1989a). Beyond Ponce de León and life satisfaction. New directions in quest of successful aging. International Journal of Behavioral Development, 12, 35-55. http://dx.doi. org/10.1177/016502548901200102

Ryff, C. (1989b). Happiness is everything, or is it? Explorations of the meaning of psychological well-being. Journal of Personality and Social Psychology, 57, 1069-1081. http://dx.doi. org/10.1037/0022-3514.57.6.1069

Ryff, C. (2014). Psychological well-being revisited: Advances in the science and practice of eudaimonia. Psychotherapy and Psychosomatics, 83, 10-28. http://dx.doi.org/10.1159/000353263

Ryff, C., \& Singer, B. (2008). Know thyself and become what you are: A eudaimonic approach to psychological well-being. Journal of Happiness Studies, 9, 13-39. http://dx.doi. org/10.1007/s10902-006-9019-0

Sandin, B., Chorot, P., Lostao, L., Joiner, T.E., Santed, M., \& Valiente, R. (1999). Positive and Negative Affect Scales (PANAS): Factorial validity and cross-cultural convergence. Psicothema, 11, 37-51.

Sanjuán, P. (2014). Promoción del bienestar psicológico: Fundamentos teóricos y aplicaciones. En P. Sanjuán y B. Rueda (eds.), Promoción y prevención de la salud desde la psicología (pp. 113-146). Madrid: Síntesis. 
Sanjuán, P., \& Magallares, A. (2014). Coping strategies as mediating variables between self-serving attributional bias and subjective well-being. Journal of Happiness Studies, 15, 443-453. http://dx.doi.org/10.1007/s10902-013-9430-2

Sanjuán, P., \& Magallares, A. (2015). Coping strategies as mediator variables between explanatory styles and depressive symptoms. Anales de Psicología, 31, 447-451. http://dx.doi. org/10.6018/analesps.31.2.173391

Schimmack, U., \& Diener, E. (1997). Affect intensity: Separating intensity and frequency in repeatedly measured affect. Journal of Personality and Social Psychology, 73, 1313-1329. http://dx.doi.org/10.1037/0022-3514.73.6.1313

Schimmack, U., Schupp, J., \& Wagner, G. (2008). The influence of environment and personality on the affective and cognitive component of subjective well-being. Social Indicators Research, 89, 41-60. http://dx.doi.org/10.1007/s11205-0079230-3

Sheldon, K., \& Elliot, A. (1999). Goal striving, need satisfaction and longitudinal well-being: The self-concordance model. Journal of Personality and Social Psychology, 76, 546-557. http://dx.doi.org/10.1037/0022-3514.76.3.482

Sheldon, K., Ryan, R., Deci, E., \& Kasser, T. (2004). The independent effects of goal contents and motives on well-being: It's both what you pursue and why you pursue it. Personality and Social Psychology Bulletin, 30, 475-486. http://dx.doi. org/10.1177/0146167203261883

Skinner, E., Edge, K., Altman, J., \& Sherwood, H. (2003). Searching for the structure of coping: A review and critique of category systems for classifying ways of coping. Psychologi- cal Bulletin, 129, 216-269. http://dx.doi.org/10.1037/00332909.129.2.216

Smith, A.L., Ntoumanis, N., \& Duda, J.L. (2007). Goal striving, goal attainment, and well-being: Adapting and testing the Self-Concordance Model in sport. Journal of Sport and Exercise Psychology, 29, 763-782.

Smith, A., Ntoumanis, N., Duda, J., \& Vansteenkiste, M. (2011). Goal striving, coping, and well-being: A prospective investigation of the Self-Concordance Model in sport. Journal of Sport and Exercise Psychology, 33, 124-145.

Watson, D., Clark, L., \& Tellegen, A. (1988). Development and validation of brief measures of positive and negative affect. The PANAS scales. Journal of Personality and Social Psychology, 54, 1063-1070. http://dx.doi.org/10.1037/00223514.54.6.1063

Wiese, S. (2007). Successful pursuit of personal goals and subjective well-being. En B. Little, K. Salmela-Aro, y S. Phillips (Eds.), Personal project pursuit: Goals, action, and human flourishing (pp. 301-328). Mahwah, NJ: Lawrence Erlbaum Associates.

World Health Organization (1948). Constitution of the World Health Organization. Disponible en http://www.searo.who. int/LinkFiles/ABOUT_SEARO_const.pdf

World Health Organization (2013). The European health report 2012: Charting the way to well-being. Copenhague: WHO.

Yi-Frazier, J., Smith, R., Vitaliano, P., Yi, J., Mai, S., Hillman, M., $\&$ Weinger, K. (2010). A Person-focused analysis of resilience resources and coping in patients with diabetes. Stress and Health, 26, 51-60. http://dx.doi.org/10.1002/smi.1258 